\title{
problèmes de sol et d'environnement en région nantaise
}

\author{
par \\ H. Gonin \\ SIMECSOL, Paris \\ D. Pfefer \\ SIMECSOL, Nantes
}

RESUME - Les études de sols ont pour premier but une synthèse de l'état du sol et du sous-sol avant une intervention humaine. Pour cela, le géotechnicien doit faire appel à différentes sciences ou techniques : la Géologie, la Géomorphologie, la Géophysique, l'Analyse structurale, l'Hydrogéologie, enfin la Mécanique des Sols. Les auteurs passent rapidement en revue ces différents aspects de l'étude de sols en présentant quelques exemples très simples recueillis parmi les études auxquelles ils ont participé dans la Région Nantaise.

\section{INTRODUCTION}

La collectivité humaine a occupé notre territoire de temps immémorial. Les données de la Géographie imposaient à l'arigine son implantation : la situation privilégiée de la Région Nantaise à cet égard est évidente, l'embouchure d'un grand fleuve donnant accès soit à la mer, soit à l'intérieur. Depuis l'explosion de la civilisation moderne, l'accroissement simultané de la population et des moyens de communication a changé à la fois la manière dont l'homme utilise un site et les raisons de son choix; les équipements collectifs occupent une place primordiale dans l'aménagement, et imposent, par la volonté humaine, des modifications parfois considérables à l'occupation du sol. Les données socio-économiques, ou les considérations politiques, pèsent lourdement sur les choix ; mais il est dangereux de méconnaitre les données naturelles et l'évolution des équilibres.Les études de sols à caractère général doivent donc d'abord se proposer de décrire l'état existant, d'étudier sa génèse, et se préoccuper des modifications que l'on envisage d'y apporter.

A travers quelques exemples simples choisis dans la Région Nantaise, nous examinons ciaprès quels sont les principaux moyens dont disposent pour cela les géotechniciens que nous sommes.

I - GEOMORPHOLOGIE, GEOLOGIE, PHOTOS AERIENNES

De tout temps, l'homme a été un observateur passionné de la nature, et il était parfois pris, devant ce qu'il ne comprenait pas, d'une terreur mystique. A l'heure actuelle, sa confiance toute récente dans les prodiges de la technique tend peut-être à l'éloigner de la patience et de la minutie de l'observation, et, au rythme accéléré de notre époque, la notion de temps n'a plus le même sens. Et pourtant, dans notre domaine, l'essentiel repose sur l'observation. Le bâtisseur se projette dans l'avenir : il lui faut à la fois tirer les leçons du passé, et prévoir les observations nécessaires pour enrichir sa connaissance.

En premier lieu, 1'histoire des sols laisse son empreinte sur eux. Les efforts tectoniques ont plissé, fracturé les terrains sédimentaires ou non et l'érosion souligne, plus qu'elle ne les masque, les accidents anciens. La végétation différencie parfois les substrats, 1'émergence des nappes se traduit dans le relief et l'occupation des sols : la Géomorphologie est l'étude des relations entre la nature des sols et la topographie. Inséparable de la Géologie, elle doit guider l'étude générale des sols.

Pour notre vieille terre, les observations des géologues sont rassemblées et publiées sous forme de cartes et de notices. C'est, bien entendu, le document de base. Mais, en zone peu urbanisée, un levé plus détaillé permet parfois d'obtenir des renseignements plus précis, notamment sur les pentes limites des affleurements, la tenue des talus naturels ou artificiels existants. Dans ce domaine précisément, la science moderne nous apporte un moyen de choix, dont la richesse, maintenant reconnue, n'est pas toujours exploitée à fond: les photos aériennes. 
Grâce aux photos aériennes, le relief peut être restitué et exagéré, à l'aide du très simple stéréoscope; l'état du terrain à différentes dates peut-être analysé. Selon les émulsions employées, les photos sont en lumière naturelle, ou utilisent des bandes du spectre électro-magnétique hors de la lumière visible : infra-rouge, ultra-violet, onde radio, radar. Nous n'insisterons pas davantage sur la photo-interprétation, qui a donné lieu à de nombreuses publications

\section{spécifiques.}

L'exemple que nous présentons ici est particulièrement simple, mais tout à fait dans le sujet du thème $n^{0}$ I. Il s'agit de la Station d'Epuration du TOUGAS, dont la construction est en cours d'achèvement dans la vallée de la Loire, sur la commune de SAINT HERBLAIN. Cette station est installée dans une zone marécageuse qui a servi de décharge publique depuis la fin de la dernière guerre (Figure 1)

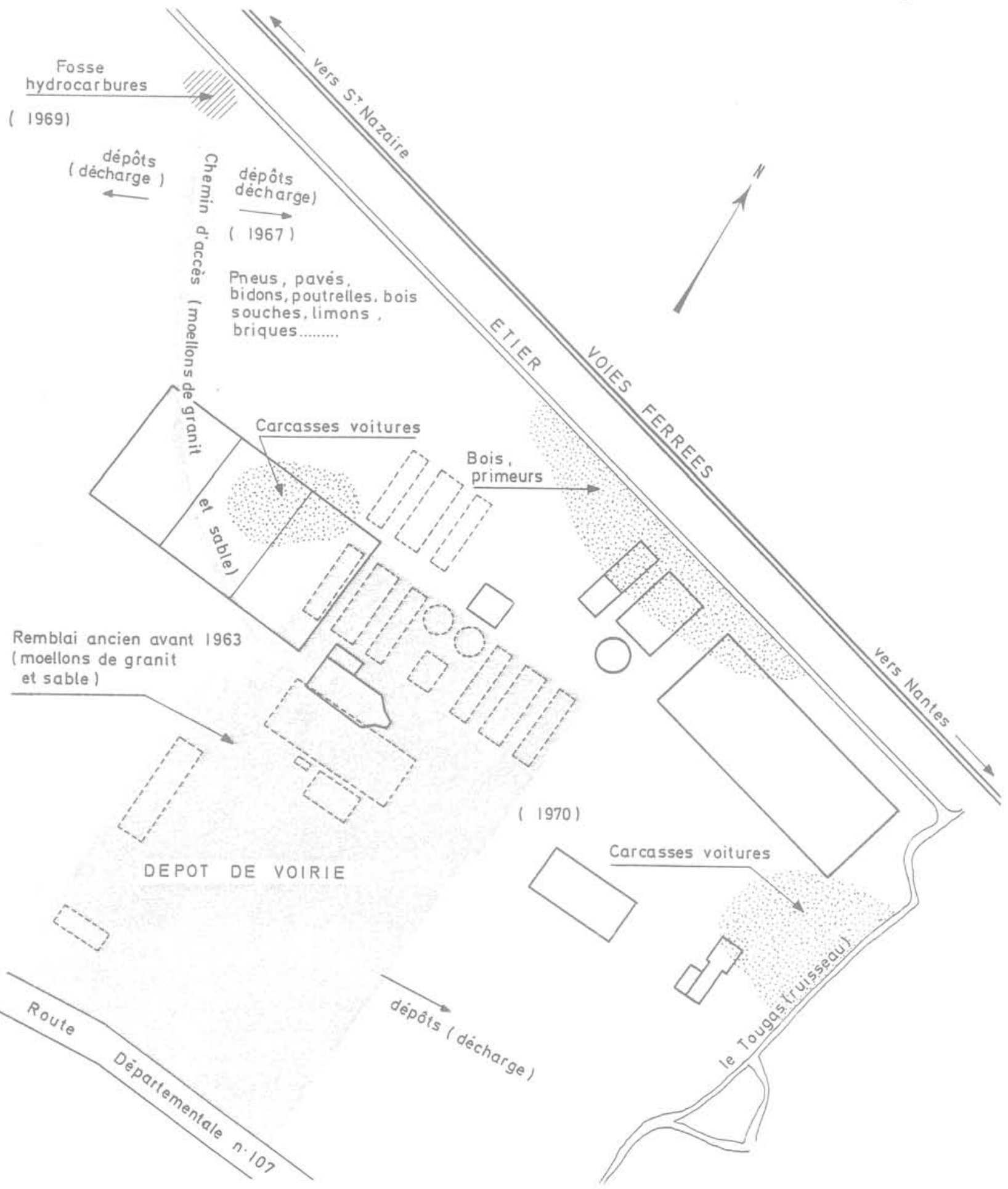

Figure 1 - Station d'Epuration de la vallée du TOUGAS - Zonage schématique - 
Une enquête complétée par l'examen des couvertures aériennes existantes a permis d'établir une carte sommaire donnant des renseignements sur la date et la nature des dépôts, préalable nécessaire à toute étude. Ces renseignements sont ici d'autant plus nécessaires que ces matériaux de décharge recouvrent des dépôts récents de la Loire en grande épaisseur, compressibles et de portance limitée, donc que les zones les plus fraichement surchargées sont les plus évolutives.

\section{II - PROCEDES GEOPHYSIQUES}

Un degré de précision supplémentaice peut, et quelquefois, doit être apporté à la connaissance du sous-sol par l'emploi de procédés géophysiques.
Le principe de la Géophysique est de mesurer en un point, ou entre deux points voisins, des grandeurs physiques dont les variations dans l'espace peuvent renseigner sur la nature, la compacité, la composition des différentes couches qui constituent le sous-sol.

La gravimétrie s'intéresse par exemple aux variations très fines de l'accélération de la pesanteur ; la sismique à la vitesse de propagation d'ondes, telles que le son; la résistivité aux caractéristiques électriques des sols. Lorsque les mesures sont faites à partir de forages (diagraphies) et non de la surface du sol, on peut également mesurer la radioactivité naturelle, l'absorption de radiations, ou, en plus, des "paramètres de forages" pendant la réalisation du trou.

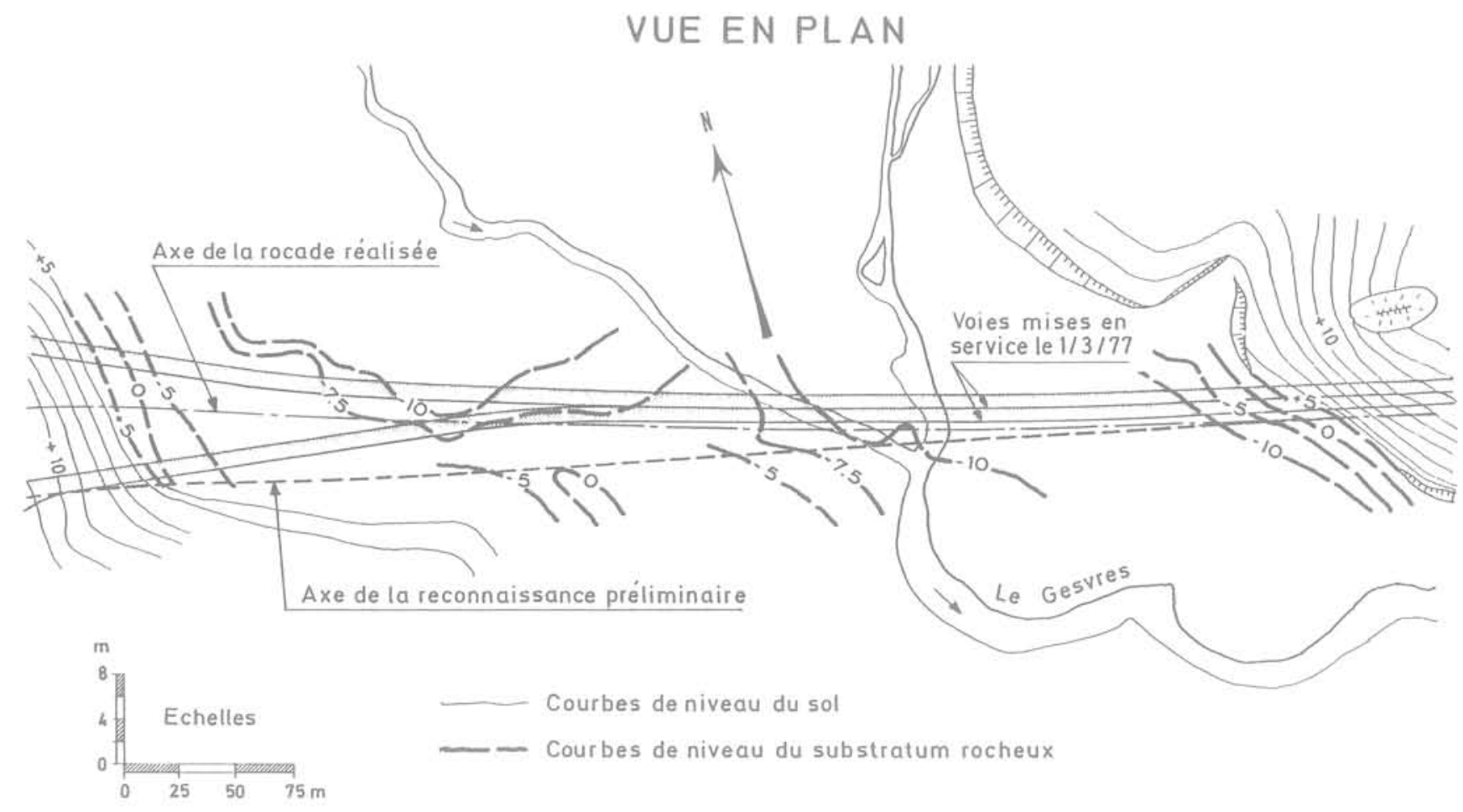

COUPE SCHEMATIQUE DES TERRAINS

Cotes

N.G.F

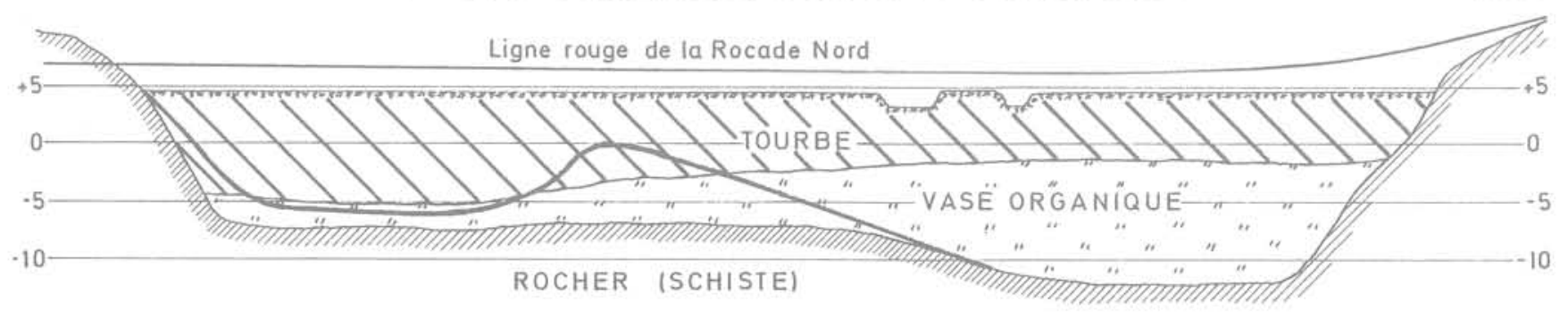

TाIIITा, Toit du rocher au droit de l'axe de la rocade réalisée

Toit du rocher suivant l'axe de la reconnaissance prèliminaire

Figure 2 - Traversée du marais de GESVRES par la rocade Nord de 1 a ville de NANTES. 
On doit toujours vérifier et étalonner par des sondages carottés les résultats des études géophysiques, ce qui conduit à demander au géophysicien d'abord en quels endroits il désire des sondages, et ensuite, une réinterprétation de sa première étude en tenant compte des résultats de ces sondages. Faute de se plier à cette exigence, des erreurs peuvent être commises.

L'exemple que nous présentons ici est négatif : il s'agit en effet d'une étude pour laquelle la liéophysique n'a pas été employée, mais il montre bien son utilité ; le procédé adapté aurait été la Sismique.

La traversée du marais de GESVRES par la rocade Nord de la Ville de NANTES a donné lieu à un projet technique dont nous avans rapporté, par ailleurs, les caractéristiques et le déroulement (KERISEL - GONIN 1978). Ce projet a fait l'objet d'un concours lancé par la Ville de NANTES en Août 1975. Les conditions du terrain étaient connues par une étude préliminaire sur un axe indiqué sur la figure 2 ; l'axe définitif du tracé retenu diffère de celui-ci sur la rive droite. On peut voir que la topographie du substratum rocheux, qui constitue une donnée essentielle pour le projet, est telle que les coupes des terrains le long de ces deux axes sont sensiblement distinctes. Le projet a do être adapté aux conditions réelles du site, imparfaitement connues au moment des études préliminaires. Il s'agit là d'un cas où une étude géophysique était rendue difficile par les conditions d'accès et des raisons d'ordre administratif ; il est néanmoins évident que seule une étude de ce type pouvait apporter sur la topographie du substratum rocheux des renseignements globaux permettant de définir un projet sans incertitude.

Ce cas est fréquent dans la Région de NANTES, où des dépôts récents de transgression masquent une topographie très variée du substratum rocheux; aussi les études récentes de déviations routières faites par la Ville de NANTES font-elles appel à la jéophysique pour définir les grandes lignes de la topographie du substratum, vérifiées par des sondages implantés en fonction des résultats de la Géophysique et des possibilités d'accès ; les études qui en résultent sont à la fois économiques et complètes ( 1 iaisons du VERTOU, de BELLEVUE). Malgré la réussite finale, la traversée routière du marais de GESVRES valait, à notre avis, d'être citée comme bien adaptée à une étude sismique qui n'a pas été faite.

\section{I - ANALYSE STRUCTURALE}

L'analyse structurale est une méthode d'étude très élaborée qui se propose de déduire les états de contraintes existant ou ayant existé dans le sous-sol ou les constructions à partir des déformations ou mouvements qui s'y manifestent ou s'y sont manifestés, et de leur symétries. Cette technique est actuellement utilisée pour les recherches pétrolières et minières. Il s'agit essentiellement de recueillir sur le terrain des observations sur les déformations, fractures ou failles des roches (au sens géologique du terme), leur distribution dans l'espace, et les mouvements relatifs des compartiments les uns par rapport aux autres. Pour suivre les évolutions, notamment d'ouvrages qui supportent des mouvements, on peut munir les deux lèvres d'une fracture de repères qui permettent des mesures tridimensionnelles.

Les trois composantes du mouvement d'une fracture sont le rejet, le décrochement et l'ouverture, suivant un système d'axe comportant l'horizontale du plan, l'horizontale qui lui est perpendiculaire et la verticale (figure 3). Pour un état donné, des organisations apparaissent qui permettent des déductions. Les évolutions récentes ou en cours peuvent être distinguées des mouvements anciens dont les traces sont "cicatrisées" (souvent par des remplissages cristallisés).

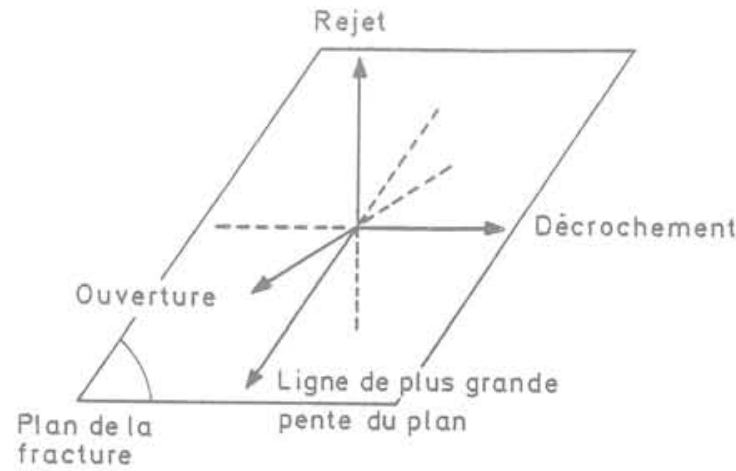

Figure 3 - Jeu d'une fracture - Définitions

Dans notre domaine, les applications de cette méthode sont plus nombreuses qu'il peut apparaftre à première vue. Nous l'avons, par exemple, utilisée avec profit, en Région Parisienne, pour l'étude du doublement du tunnel de SAINT CLOUD, dans l'analyse de la stabilité de la bordure Est de la colline du TROCADERO et de la fissuration du tunnel existant. Elle s'applique à l'étude des mouvements d'une structure fissurée, à celle de la stabilité de talus rocheux. L'exemple très simple que nous avons choisi comme illustration est celui de l'étude, en Juin 1979, de la stabilité d'une passerelle pour piétons, à la pointe de CHEMOULIN à SAINT NAZAIRE.

Le relevé des discontinuités de la roche (il s'agissait là d'un gneiss) est reporté sur la figure 4 en projection stéréographique et en représentation polaire, qui permet l'établissement d'un diagramme statistique.

Cette étude permet de déterminer les risques d'instabilité, et de proposer un confortement par boulons d'ancrage (cf. figure 5). La confortation de ce morceau de falaise et l'établissement, avec une notable économie de moyens, d'un chemin piétonnier ne constituent ils pas un bon exemple du respect de l'environnement? 
DIAGRAMME DE SCHMIDT demi-sphère infèrieure

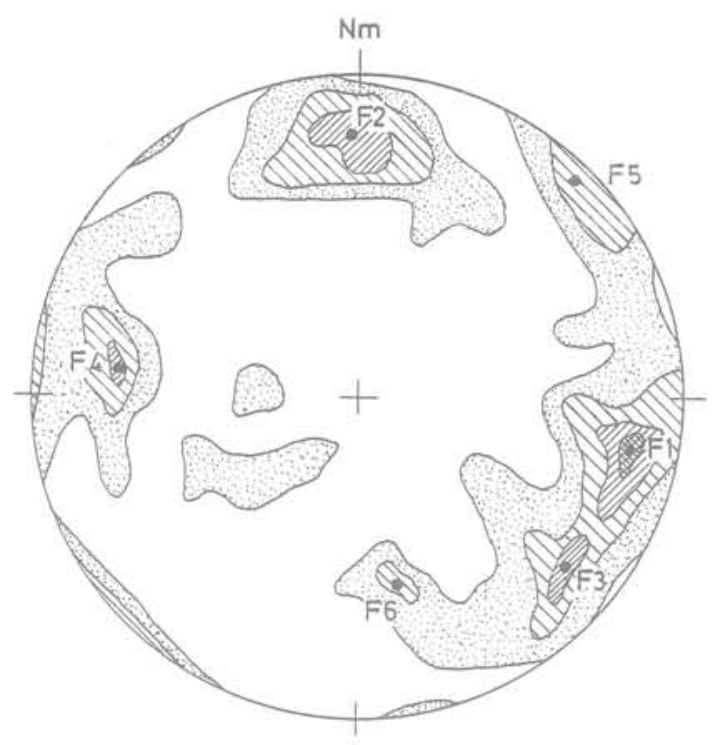

Densité des mesures

$$
0.5<u<1.5
$$

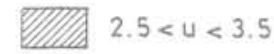

DII $1.5<u<2.5$

$3.5<4$

Figure 4 - Pointe de CHEMOULIN

Diagramme polaire statistique des discontinuités.

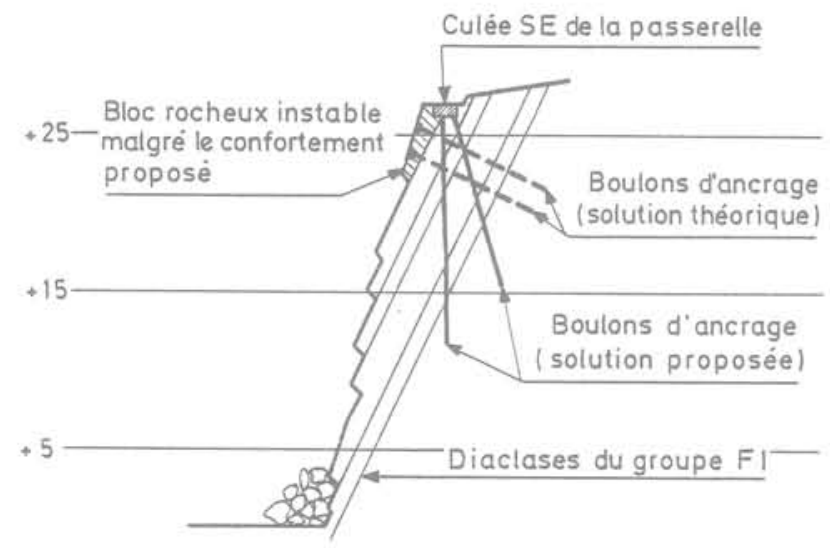

Figure 5 - Passerelle pour piétons de la Pointe de CHEMOULIN

Confortation de l'appui de la culée.

\section{IV - HYDROGEOLOGIE}

L'Hydrogéologie nécessite des études longues et rassemblant de multiples observations sur la pluviométrie, les bassins versants, la piézométrie des nappes, les écoulements des eaux vives et souterraines. Il est très important de s'en préoccuper, car les équipements collectifs, qui trouvent souvent une implantation privilégiée dans le lit majeur des fleuves, apportent des modifications au régime des eaux, sans parler des problèmes liés à la pollution des nappes. Ces études sont d'autant plus nécessaires que l'eau est une ressource naturelle dont l'homme a grand besoin ; mais on ne doit pas perdre de vue que l'exploitation des ressources en eau a souvent des incidences sur les travaux de construction.

Nous ne nous référerons pas ici à une étude particulière, mais passerons en revue quelques problèmes qui se présentent fréquemment dans la Région Nantaise et qui sont en relation avec I'Hydrogéologie.

En premier lieu, on oublie souvent que le contexte géologique, dans la vallée de la Loire et ses affluents, est favorable à l'existence de conditions artésiennes au contact du substratum rocheux. L'existence de ces conditions faiblement artésiennes est souvent très difficile à mettre en évidence d'une manière simple, rapide et peu coateuse; elle conditionne cependant l'équilibre et la consolidation des dépôts récents de transgression qui forment le remplissage alluvial. Malheureusement, il arrive souvent que dans les projets on doive se contenter sur ce point de choisir une hypothèse sans certitude absolue, faute de temps et d'argent.

Par ailleurs, c'est un lieu commun de dire qu'il faut se préoccuper de l'incidence des abaissements phréatiques dos aux épuisements dans les fouilles, à l'extérieur de cellesci. Le problème se pose avec une acuité particulière pour les terrains que forment le remplissage alluvial de la vallée de la Loire, constitué d'une succession de lits sableux et de fines passées de vase marine. La grande distorsion des perméabilités qui en résulte a pour conséquence d'étendre au loin le rayon d'influence d'un abaissement phréatique local.

Enfin, la pollution des eaux souterraines est un facteur dont il faut tenir compte dans les projets. La pollution par des hydrocarbures, et notamment des essences léqères, présente des dangers et nécessite des précautions sur lesquels il n'est pas inutile d'insister. Nous devons en tenir compte dans les études de sols.

\section{V - ET LA MECANIQUE DES SOLS?}

Etude du comportement mécanique des sols, elle doit intervenir, au stade des études générales, pour éclairer ou confirmer toutes les observations recueillies et exploitées. 
Trop souvent, elle est considérée comme un but en soi, avec des formules passe-partout et des recettes hermétiques au profane, Nous concevons pour notre part l'utilisation de nos connaissances en Mécanique des Sols comme un outil au service d'une recherche inductive portant sur les problèmes qui se posent et l'examen des solutions qui peuvent y être apportées. C'est pourquoi, à notre sens, l'étude de Mécanique des Sols doit se fixer des buts précis, avec la meilleure économie de moyens possible; et elle n'est qu'une part, essentielle mais limitée, de l'étude des sols à caractère général. Ce sera par exemple, la détermination des caractéristiques à long terme d'une argile dont l'origine géologique sera bien précisée, ou de la compressibilité et des facteurs de la résistance au cisaillement d'un dépôt récent parfaitement identifié, ou l'étude granulométrique d'alluvions fluviatiles, ou des caractéristiques de gonflement d'une marne formant une couche sédimentaire bien localisée.

\section{CONCLUSIONS}

Comment doit être présentée l'étude de sols? A quoi peut elle servir? On permettra aux auteurs, en conclusion, de donner leurs idées personnelles sur cette question.

La synthèse des études faites doit être présentée clairement dans un rapport court. Si. ce rapport a plus de vingt pages, il ne sera pas lu dans son intégralité. Au besoin, on peut renvoyer à des annexes plus détaillées traitant d'une matière particulière.

Il doit être accompagné de plans et de schémas synthétiques peu nombreux, établis dans un souci de clarté et dans un but didactique. On ne doit pas hésiter à donner des explications d'ordre général, en évitant toutefois les stéréotypes, ce qui demande un effort constamment renouvelé. Ce rapport doit envisager les répercussions lointaines des aménagements prévus, et donner au lecteur le désir de s'en préoccuper, en suscitant sa curiosité. Il doit souligner les avantages ou les inconvénients des différentes solutions envisageables, leurs conséquences prévisibles, mais s'il peut orienter un choix, il ne doit pas chercher à l'imposer.

Enfin, les prévisions doivent pouvoir être comparées aux réalités. Le rapport suggérera la mise en place d'un système de mesures et de cantrôles adaptés aux problèmes qui se posent, et insistera sur les dispositions à prendre pour en assurer le suivi. Il y a là, dans la pratique, un problème d'une très grande difficulté. Faute d'un effort collectif suffisant pour recueillir des données, car chacun estime que cet effort incombe au voisin plus qu'à lui-même, nous sommes mal armés pour résoudre au mieux des problèmes qui se sont déjà posés. Il y a parfois lieu d'être consterné, lorsqu'un sinistre arrive, en voyant le peu de renseignements dont les experts disposent pour en rechercher les causes. Quelques observations consignés en leur temps seraient souvent d'une aide extrêmement précieuse, et les cas ne sont pas rares ou cela aurait permis de prévoir des incidents et de prendre préventivement des mesures adaptées.

L'un de nous s'étonnait un jour, devant les questions curieuses et pertinentes qui lui étaient posées par son client, que celui-ci n'ait que très superficiellement lu le rapport d'étude de sols qu'il avait entre les mains. "Vous savez bien, Monsieur, que les rapports sont faits pour ne pas être lus", fut la réponse en forme de constat humoristique.

Faisons au moins en sorte que nos rapports vaillent la peine d'être lus !

\section{REFERENCES}

DE SLOOVERE P, (1980) Reconnaissance du sous-sol par enregistrement instantané de divers parametres au cours de la perforation d'un forage destructif.

Bulletin de l'Association internationale de Géologie de 1'Ingénieur, (21) 43-48.

HOEK E, (1974) Rock slope Engineering, the institution of mining and mettalurgy, London.

KERISEL J, GONIN H, (1978), La traversée du marais de Gesvres par la rocade Nord de la ville de Nantes.

Revue Générale des Routes et Aérodromes, (543), 45-58.

L'évaluation Géotechnique et la Télédétection appliquéesaux projets routiers dans les pays en voie de développement (1980).

Bulletin de liaison des Laboratoires des Ponts et Chaussées, (107) 37-72.

LoUIS C, (1977), Apport de la Géotechnique aux exploitations minières à ciel ouvert, B.R.G.M. Orléans. 